\title{
El poder educativo de lo inesperado. Estudio de experiencias innovadoras en la universidad
}

\author{
The educational power of the unexpected. \\ Study of innovative experiences in the university
}

ISSN 1510-2432 - ISSN 1688-9304 (en línea) - DOI: https://doi.org/10.18861/cied.2016.7.2.2609

María Fernanda Melgar

Doctora en Psicología, Universidad Nacional de San Luis, Argentina. Máster en Psicología de la Educación, Universidad de Murcia, España. Licenciada en Psicopedagogía, Universidad Nacional de Río Cuarto, Argentina. Becaria posdoctoral, Consejo Nacional de Investigaciones Científicas y Técnicas. Profesora Ayudante 1.a, Facultad de Ciencias Humanas, Universidad Nacional de Río Cuarto. Autora de artículos científicos sobre museos y educación, museos y creatividad, museos y TIC.

Romina Elisondo

Doctora en Psicología, Universidad Nacional de San Luis, Argentina y Universidad de Murcia, España. Magíster en Educacióny Licenciada en Psicopedagogía Universidad Nacional de Río Cuarto, Argentina. Investigadora asistente, Consejo Nacional de Investigaciones Científicas y Técnicas, Argentina.. Profesora adjunta, Facultad de Ciencias Humanas, Universidad Nacional de Río Cuarto. Autora de libros, capítulos y artículos científicos vinculados a temas de creatividad y educación.

Danilo Donolo

Doctor en Ciencias de la Educación, Universidad Católica de Córdoba, Argentina. Profesor consultor, Facultad de Ciencias Humanas, Universidad Nacional de Río Cuarto, Argentina. Investigador Independiente, Consejo Nacional de Investigaciones Científicas y Técnicas, Argentina. Autor de libros, capítulos y artículos científicos sobre inteligencia, creatividad, educación, motivación y TIC, entre otros.

\section{Rodolfo Stoll}

Ingeniero Electricista, Universidad Nacional de Río Cuarto, Argentina. Integrante, equipo de investigación, Grupo de Energía Solar (GES). Profesor, Facultad de Ingeniería, Universidad Nacional de Río Cuarto. Autor de artículos científicos sobre energías renovables.

Fecha de recibido: 03/03/2016

Fecha de aceptado: 22/06/2016

\section{Resumen}

Aumentar, invertir, investir, abrir y lograr una indisciplina óptima son cinco ideas que proponemos para generar contextos educativos creativos. Destacamos el papel de lo inesperado y de la sorpresa como elementos necesarios en las innovaciones educativas. En el artículo presentamos un estudio sobre experiencias realizadas con docentes inesperados en el contexto de clases de psicopedagogía en la Universidad Nacional de Río Cuarto (Argentina). Para la recolección de datos empleamos narrativas y cuestionarios abiertos de respuesta individual. Compartimos las valoraciones realizadas por los estudiantes y docentes que participaron del estudio. Los estudiantes valoraron la experiencia positivamente destacando aspectos como lo inesperado de la propuesta, la experimentación y el trabajo grupal. Los docentes destacaron el desafío que supone 
compartir el conocimiento y generar nuevas oportunidades para aprender. A partir de los datos analizados se presenta una nueva clasificación de docentes inesperados: docentes invitados, no esperados por los estudiantes, y docentes esperados que se desempeñan de manera inesperada. Invitar a docentes y desarrollar intervenciones didácticas inesperadas son acciones propicias para la construcción de contextos creativos en la universidad.

Palabras clave: creatividad, innovación educativa, enseñanza universitaria, aprendizaje creativo.

\begin{abstract}
Increasing, inverting, investing, opening and reaching optimal indiscipline are five ideas that we propose to generate creative educational contexts. We emphasize the role of the unexpected and of surprise as necessary elements in educational innovation experiences. We present a study of experiences with unexpected teachers in educational psychology classes at the National University of Río Cuarto (Argentina). For data collection narratives and open questionnaires with individual answers were used. We share assessments made by students and teachers who participated in the study. Students rated the experience positively, highlighting aspects such as the unexpected character of the proposal, experimentation and group work. Teachers emphasized the challenge it meant sharing knowledge and creating new opportunities of learning. From the data analyzed, a new classification of unexpected teachers is presented: invited teachers not expected by the students, and expected teachers that do unexpected things. Inviting teachers and developing unexpected educational interventions are actions conducive to building creative contexts at University.
\end{abstract}

Keywords: creativity, educational innovation, university teaching, creative learning.

\title{
1. Introducción
}

Hace unos años los autores del presente trabajo relataron experiencias educativas con docentes inesperados, es decir, clases y propuestas de enseñanza con personas ajenas a las cátedras, docentes que no deberían haber estado en ese momento y en ese lugar, profesores, investigadores y especialistas que no son esperados y sorprenden con su visita (Elisondo, Donolo y Rinaudo, 2013). La relación entre los docentes inesperados y la creatividad es compleja y se plantea como posibilidad y desafío. La inclusión de docentes inesperados no necesariamente promueve la creatividad de los estudiantes, no obstante amplía de manera significativa las posibilidades de interacción con especialistas, conocimientos diversos y procedimientos alternativos. Especialistas, conocimientos y procedimientos nuevos son componentes indispensables para la construcción de contextos educativos orientados hacia la creatividad (Rinaudo y Donolo, 2000; Davies et al., 2013; Beghetto y Kaufman, 2014).

En el presente escrito se analizan significados y valoraciones de los estudiantes y profesores que participaron de innovaciones donde se incluyeron docentes inesperados en las aulas. Las experiencias desarrolladas se consideran innovaciones áulicas en tanto suponen prácticas que alteran el sistema de relaciones unidireccionales que caracteriza a la clase tradicional y rompen con el statu quo y la dinámica de las aulas (Lucarelli, 2009). Se presenta un estudio cualitativo exploratorio de indagación de significados y valoraciones respecto de innovaciones educativas. Se destaca la importancia de investigar las innovaciones, los impactos que producen y los significados que construyen los actores respecto de los procesos de cambio. Coincidimos con Pizzolito y Macchiarola (2015) en la necesidad de articular los procesos de producción de conocimiento y mejoramiento de la enseñanza, integrando investigación e innovación educativas.

En el artículo se amplía la definición inicial de docentes inesperados y se construyen dos 
tipologías: docentes no esperados y docentes esperados que hacen cosas inesperadas. Previo a la narración de las experiencias se presenta la base teórica acerca de la educación, la creatividad y el poder educativo de lo inesperado. Luego se describe el contexto en el que se desarrollaron las experiencias, las personas que participaron, la estructura de la clase y los objetivos educativos propuestos. Con el propósito de conocer significados y valoraciones de los participantes se utilizaron cuestionarios de opción abierta y narrativa. Los resultados se analizaron de manera cualitativa. Una vez presentados los resultados y las interpretaciones, se proponen las consideraciones finales.

\section{Perspectivas teóricas}

Se presentan dos perspectivas sobre la creatividad relacionadas con la educación y con los avances en neurociencias.

\subsection{Creatividad y educación}

En un artículo reciente Elisondo (2015) plantea cinco ideas desde el punto de vista de las investigaciones sobre creatividad para la construcción de contextos creativos de aprendizaje. Pensar la educación desde una perspectiva creativa implica divergencias, flexibilidad y alternativas a la hora de diseñar las propuestas pedagógicas, los contenidos y los encuadres didácticos.

La primera idea es aumentar la educación, es decir, ofrecer posibilidades ilimitadas e inesperadas para aprender en interacción con los otros y con las tecnologías. Aumentar la educación supone no poner límites, no pensar "no van a poder", "esto no es para ellos", "nunca lo van a entender". Aumentar la educación es cambiar de perspectiva, es pensar en las posibilidades de aprendizaje y no en las limitaciones o supuestas limitaciones de las personas. Desde una perspectiva sociocultural de la educación, Bruner (1997) propone una serie de postulados que permiten diseñar procesos educativos. En este sentido, aumentar la educación también supone ampliar los límites. Para Bruner, la posibilidad de acceder a los significados de la cultura está doblemente limitada en las personas: primero, por la propia naturaleza del funcionamiento mental humano y, segundo, por las constricciones impuestas por los sistemas simbólicos accesibles a las mentes humanas (principalmente por la propia naturaleza del lenguaje). Una posible implicación educativa de este postulado se vincula con las oportunidades, situaciones, organización de entornos, tareas de aprendizaje, materiales bibliográficos, uso de TIC e interacciones con otros (sujetos e instituciones) que la educación formal pueda estar ofreciendo para que se reduzca la brecha de esos límites en los sujetos (Melgar, Donolo y Gómez, 2009).

Una segunda idea es invertir en educación. Invertir en educación significa, para los autores del presente trabajo, cambiar de posición, dar vuelta, desestructurar algunas estructuras y convenciones de los contextos educativos. Asimismo, significa apostar, arriesgar y asumir los riesgos de generar innovaciones educativas. Esta inversión supone esfuerzos, dedicación y trabajo. Las innovaciones en el campo educativo no suceden de un día para el otro sino que requieren de inversiones importantes de los sujetos implicados, quienes deben invertir tiempo, energía y pasión en la construcción de nuevos contextos para aprender y enseñar. Dar vuelta a la educación implica repensar tiempos, espacios, roles, contenidos y mediciones en los entornos educativos. Es invertir los tiempos de trabajo en la escuela y en el hogar tal como proponen las iniciativas de clases invertidas pero también, y fundamentalmente, implica construir nuevas perspectivas respecto del rol de docentes y estudiantes, los espacios de encuentro, las mediciones tecnológicas y los procesos de adquisición y producción de conocimientos. 
La tercera idea es investir la educación. Las nuevas perspectivas educativas deberán atender a aspectos subjetivos implicados en todo aprendizaje humano. Parece indispensable hacer circular el deseo en los encuentros educativos y reconocer la potencia creativa de la alegría (Fernández, 2013). Las nuevas perspectivas educativas deberán atender a los deseos y emociones que habitan las aulas y las instituciones, propiciando entornos donde se construyan deseos de conocer y procesos de autoría de pensamientos y palabras.

Bruner (1997) sostiene que la educación desempeña un papel fundamental en la formación del yo, en términos de agencia y estima. La agencia se vincula a la percepción de sentir que podemos emprender y llevar a cabo actividades por nuestra cuenta; y la estima supone una valoración de nuestra eficacia en llevar a cabo lo que esperábamos o lo que se nos indicó hacer. En este sentido, tanto una como otra suelen verse afectadas por la disponibilidad de apoyos que se ofrecen desde fuera. Las prácticas educativas ordinarias, aun en el nivel superior, deben examinarse desde la perspectiva de las contribuciones que hacen a estos dos aspectos del yo. Fomentar la agencia y estima en los estudiantes es investir la educación.

La cuarta idea es abrir la educación. Salir de las aulas, tal como proponen Melgar y Donolo (2011) y Badilla (2007), es ofrecer oportunidades de aprender en contextos formales, no formales e informales, es expandir la educación, descentralizar el aprendizaje y promover la educación permanente como valor educativo necesario. Aprender más allá de las fronteras de las aulas es también propiciar la creatividad en tanto se amplían significativamente los horizontes conceptuales y las interacciones con otras personas. Salir de las aulas, visitar otros contextos, reales o virtuales es construir nuevas relaciones y vivir nuevas experiencias de aprendizaje y creación de conocimientos. Por ejemplo, los museos permiten salir de las aulas en busca de conocimientos, experiencias, objetos y relaciones interesantes para aprender y crear, tal como lo sostienen Melgar y Donolo (2011).

La quinta idea es generar una indisciplina óptima. Para la construcción de contextos creativos es necesario romper con algunas rutinas que dificultan las innovaciones y, asimismo, desarrollar un trabajo indisciplinado con los conocimientos, salirse de las fronteras disciplinarias y construir contenidos indisciplinados para aprender y enseñar. Es indispensable cierta indisciplina óptima que permita la creatividad y la innovación sin apartarse de valores y normas éticas esenciales para el desarrollo social, educativo y personal. La indisciplina que se propone es instituyente y se orienta hacia la innovación, respetando valores y acuerdos sociales fundamentales para la formación de las personas.

En definitiva, las cinco ideas propuestas pretenden generar contextos educativos poderosos. Según Rinaudo (2014) estos contextos promueven el aprendizaje activo y constructivo en el marco de situaciones reales, estimulan la participación de las personas y propician oportunidades para trabajar activamente en el logro de nuevos conocimientos. Los contextos poderosos consideran la calidad del conocimiento, los tipos de lenguaje, discursos y herramientas que circulan, los procesos de retroalimentación, las oportunidades para la colaboración entre pares y el carácter de las evaluaciones.

Las ideas también se relacionan con lo poderoso de realizar cambios y animarse a innovar, apostando a la transformación educativa. Las instituciones cuentan con numerosas rutinas que son necesarias y saludables para nuestro cerebro, pero que provocan acostumbramientos que dificultan el cambio. Lo inesperado rompe con la rutina generando nuevas sensaciones o enriqueciendo las vivencias que tenemos. 


\subsection{Creatividad y neurociencias}

Desde la perspectiva de las neurociencias Ballarini (2015) sostiene que la novedad tiene un papel clave en el proceso de aprendizaje, a tal punto que sin el efecto de la novedad muchas memorias jamás se consolidarían y se perderían por siempre en el olvido. Una experiencia novedosa con relevancia pedagógica desarrollada durante el horario regular de la escuela puede mejorar la memoria de los diferentes aprendizajes realizados en ese contexto temporal de un par de horas previas o posteriores. En situaciones experimentales (Ballarini et al., 2013; 2009) se ha observado que los eventos novedosos disparan la síntesis de proteínas y etiquetan conexiones sinápticas: las proteínas producto de la novedad lograrían sostener dicha conexión de forma duradera. La novedad suele estar ligada a la curiosidad. Ser curioso está ligado a lo desconocido, a la noción de no tener idea de lo que sucede, de lo que está por venir. Cuando una situación excede nuestro conocimiento, cuando nos sorprende, es posible que se genere el etiquetado conductual, es decir, un mecanismo fisiológico que nos permite guardar de forma segura y perdurable la información.

Los desarrollos de Manes (2014) sobre neuroplasticidad, creatividad y relevancia de la interacción social en la construcción de conocimientos también ofrecen interesantes herramientas para la innovación educativa. Las neurociencias nos invitan y desafían a generar ambientes de enseñanza y aprendizaje que consideren las infinitas posibilidades del cerebro humano.

Desde las teorías de la creatividad, lo inesperado también cobra especial interés. Los procesos creativos tienen múltiples componentes inesperados, sorpresivos y novedosos. Inesperadas son las conexiones que se construyen durante los insight. También son inesperados los resultados e impactos de los procesos de creación y producciones creativas. Los creativos también surgen en contextos y momentos inesperados: es difícil predecir cuándo y dónde aparecerá un creativo que romperá con las estructuras de lo establecido. También son inesperadas las reacciones de los demás ante dichas rupturas. Los procesos creativos en sus diferentes etapas y los productos emergentes siempre incluyen aspectos novedosos, originales y alternativos. Las diferentes teorías y concepciones de la creatividad reconocen componentes inesperados y novedosos en los procesos creativos, tanto las perspectivas de formulación como de resolución de problemas (Kozbelt, Beghetto y Runco, 2010).

En la intersección entre creatividad y educación, lo inesperado puede ser una interesante propuesta para la innovación educativa (Beghetto y Kaufman, 2014; Elisondo, Donolo y Rinaudo, 2013). Los contextos educativos inesperados ofrecen múltiples posibilidades de generación de ideas y promueven emociones positivas y relaciones de colaboración entre los participantes. Un análisis de investigaciones permite identificar experiencias vinculadas al diseño de contextos educativos con objetos excéntricos inesperados (Elisondo, Rinaudo y Donolo, 2011), propuestas con docentes inesperados (Elisondo, Donolo y Rinaudo, 2012), propuestas educativas en lugares inesperados (Elisondo y Donolo, 2013; 2014; Elisondo y Melgar, 2015) y evaluaciones que rompen con la manera tradicional de valorar los aprendizajes en la universidad (Elisondo y Donolo, 2015).

En un estudio reciente investigamos valoraciones de los estudiantes respecto del cursado de asignaturas donde se incluyen innovaciones (Elisondo y Melgar, 2016). Se observó que el $90 \%$ de los estudiantes encuestados definen a las propuestas inesperadas como las actividades de la materia que más les agradaron. Asimismo, las experiencias inesperadas son las más recordadas por los estudiantes al finalizar el cursado de las asignaturas. Por 
su fuerte componente novedoso las actividades inesperadas parecen haber quedado guardadas en la memoria y ser recuperadas al momento de reflexionar acerca de lo realizado durante el cursado de toda la materia. Lo agradable de las actividades y la carga emocional positiva de las mismas también han facilitado el recuerdo y la recuperación al finalizar el cuatrimestre.

Desde múltiples aristas -las neurociencias, las teorías de la creatividad y la educaciónse observa que las sorpresas, lo novedoso y lo inesperado promueven el recuerdo, el aprendizaje y la vivencia de experiencias y emociones significativas. Siguiendo estas líneas argumentativas proponemos experiencias educativas con docentes inesperados, experiencias que además de ser novedosas promueven interacciones con otras personas y conocimientos, interacciones que desde las perspectivas socioculturales de la educación son fundamentales.

\section{Estudio exploratorio de experiencias innovadoras}

\subsection{Decisiones metodológicas}

Se desarrolló un estudio exploratorio para el cual se adoptó una perspectiva cualitativa con el fin de comprender las significaciones y valoraciones construidas por los actores que participaron de las experiencias innovadoras. Participaron del estudio 35 estudiantes de quinto año de la carrera de Licenciatura en Psicopedagogía y dos docentes: el profesor invitado y la profesora que propuso la actividad. Se utilizaron dos instrumentos: cuestionario con preguntas abiertas para estudiantes y narrativas para docentes. El cuestionario incluía preguntas sobre la organización de las actividades, los contenidos, las relaciones con otros contenidos de la materia, los impactos en la formación profesional y valoraciones generales de la propuesta. Se solicitó a los docentes que redactaran una narrativa considerando expectativas, emociones y vivencias experimentadas en los diferentes momentos de la propuesta, preparación de las clases, desarrollo y evaluación posterior.

Las respuestas de los estudiantes y las narrativas de los docentes se analizaron de manera cualitativa mediante métodos de comparaciones constantes. Se construyeron categorías tanto para el análisis de las voces de los estudiantes como para las narrativas de los docentes considerando valoraciones, experiencias y emociones emergentes.

\subsection{Descripción de las experiencias}

Las experiencias se realizaron con estudiantes de la cátedra de Psicometría Educacional. La actividad tuvo por objetivo reflexionar sobre la medición en Psicología y Educación a partir de una experiencia alternativa.

Convocamos a un profesor de la Facultad de Ingeniería de la UNRC para pensar en una serie de experimentos. Los conceptos de la materia fueron una excusa para ofrecer a los estudiantes la posibilidad de escuchar a un profesional proveniente de otra área y enriquecernos culturalmente con sus aportes sobre la medición de la energía, la producción y consumo en nuestros hogares.

Llevó alrededor de dos meses el diseño de la actividad: encontrar los experimentos, buscar los materiales y dar sentido y significación a los conceptos. Animarnos a invertir en educación no fue tarea simple. Fue necesario desarmar ciertas ideas sobre qué hacer en una clase de psicometría. 
Luego de varios intercambios decidimos junto con el profesor invitado que los conceptos de potencia y energía eran dos buenos ejemplos para comprender las mediciones que solemos realizar en nuestra vida cotidiana, a partir de indicadores. Estos dos conceptos nos permitirían realizar comparaciones sobre mediciones en psicología.

En definitiva, medir es determinar el valor de algo por comparación con una unidad establecida que se toma como referencia, generalmente mediante un instrumento graduado con dicha unidad. Medir encierra un proceso de cuantificación que supone, al menos, dos operaciones: definir las características que se quieren medir y construir una escala para medirlas.

Las experiencias se desarrollaron en un aula diferente a la utilizada en el dictado de la materia y en el transcurso de dos jornadas. El primer encuentro tuvo una duración de dos horas y el segundo de una hora. Durante el primer encuentro se presentó al docente inesperado, un ingeniero. Luego, durante unos 30 minutos expuso su marco referencial, planteó una serie de problemas desde su perspectiva de la ingeniería y se detuvo en los conceptos de energía y potencia como aspectos clave para realizar mediciones. Más tarde comenzaron las actividades prácticas, vale decir, los juegos y experimentos. Los estudiantes se dividieron en grupos al azar. Cada grupo tenía un tiempo determinado para resolver un experimento y seguir el circuito.

El primer experimento del circuito se denominó ¡Se hizo la luz! Los materiales empleados fueron veladores y focos de diferentes vatios. Los estudiantes debían responder ¿cuál era el foco de más potencia? y ¿cómo se daban cuenta? También se mencionaba que podían formular otras preguntas si lo deseaban. Para poder responder a estos interrogantes trabajaron con conceptos tales como indicadores, unidades de medida y otros.

El segundo experimento se denominó ¡Se me vuelan las ideas! Los materiales fueron dos ventiladores de diferentes potencias. Los estudiantes debían responder ¿cuál era el ventilador de mayor potencia? y ¿cómo se dieron cuenta? Asimismo, teniendo en cuenta los conceptos de energía y potencia se promovía la formulación de otras preguntas.

El tercer experimento se denominó ¡iResistiré!! Los materiales empleados fueron dos resistencias de agua para termo, dos bols y un medidor. Los interrogantes a responder fueron ¿cuál de las dos resistencias tiene más potencia? y ¿cómo se dieron cuenta? Se sugería que empleando los conceptos de energía y potencia formularan otras preguntas.

El cuarto experimento se denominó ¡Se hizo la luz! Segunda Parte. Los materiales empleados fueron dos linternas con dinamo. Los interrogantes a resolver fueron ¿de dónde saca la linterna la energía para que funcione el foco? y ¿de qué depende la intensidad? Teniendo en cuenta los conceptos de energía y potencia se les solicitó formular otras preguntas si así lo deseaban.

\section{Análisis de resultados}

El análisis de los resultados fue realizado considerando la perspectiva de los estudiantes y de los docentes.

\subsection{Perspectiva de los estudiantes}

Los autores del presente trabajo comentaron las valoraciones de los estudiantes acerca de cómo piensan que esta experiencia contribuyó en su formación profesional, qué fue lo que les gustó y qué modificarían de la experiencia. 


\section{Contribución a la formación profesional}

En relación a este punto los estudiantes destacaron que la propuesta les permitió reflexionar sobre medición en psicología desde otra disciplina, además de enriquecer su cultura general, vivenciar una clase distinta y ampliar la mirada y conocer desde otras perspectivas.

En este tipo de respuestas mencionaron que a través de los experimentos pudieron pensar sobre los test y sus usos. Compartimos la respuesta de un estudiante que permite visualizar este aspecto.

"Considero que una experiencia como esta contribuye en nuestra formación profesional en el sentido de ayudarnos a mejorar nuestro punto de vista respecto a la medición, y a poner en práctica elementos de la teoría y de la vida cotidiana para poder medir lo que nos interese saber sobre una variable específica (JP)".

La experiencia sirvió para enriquecer su cultura general sobre saberes cotidianos. En este tipo de respuesta los estudiantes mencionaron aspectos tales como reflexionar sobre aspectos de la vida cotidiana -cosas simples- y adquirir nueva información sobre cosas que desconocían. La respuesta de uno de los estudiantes permite ilustrar el aspecto mencionado.

“[...] Más allá de que nos sirva en nuestro futuro profesional, a su vez esta experiencia nos ayudó en saberes de la vida cotidiana (GG)".

Asimismo, señalaron que la experiencia permitió vivenciar una clase distinta saliendo de la estructura típica. En este tipo de respuesta, lo distinto se vincula a la visita de un profesional de otra área, a la posibilidad de trabajar en grupos, de escuchar sobre una temática diferente a la formación profesional, de realizar juegos y experimentos, entre otros. A continuación la respuesta de un estudiante permite ilustrar la categoría mencionada.

“Fue una experiencia diferente porque salió de la estructura de la rutina de la formación que hasta el momento hemos recibido. Fue una clase creativa en donde teníamos que explorar cosas nuevas, en donde nos permitió escuchar y discutir otro punto de vista, de otra persona [...] (FM)".

Otra contribución fue que la experiencia les permitió ampliar la mirada y conocer desde otras perspectivas. Se destacaron aspectos tales como que la experiencia les hizo ver que existen otros aspectos de la realidad más allá de los estudiados en la carrera, que les permitió abrir la mente hacia la indagación, a no cerrarse y hacer siempre lo mismo. Se destacan a continuación las palabras de un estudiante, que permiten ilustrar el aspecto mencionado.

“Contribuye de diversas maneras. Primero porque la parte introductoria de la actividad fue muy amplia, muy abarcativa y nos permitió acceder a conocimientos muy interesantes que quizá durante la carrera no vemos porque precisamente profesionalmente no nos competen. También creo que la parte de la actividad práctica nos demostró nuevamente que hay variadas formas de enseñar contenidos y que cuenta tener creatividad. Y la parte de sacar la energía que consumimos nos sirvió para concientizarnos. También fue una actividad distendida, en la que hubo buen clima de trabajo y nos sacó de las clases rutinarias (LM)". 


\section{Aspectos destacados de las experiencias}

Se destacaron los siguientes aspectos: experimentar con objetos, ampliar los conocimientos generales sobre el consumo de energía, hacer algo distinto que rompiera con la rutina y trabajar en grupos.

En relación con el primer aspecto, los estudiantes mencionaron que experimentar con objetos resultó novedoso para la formación. En este sentido, comentaron que no suele ser habitual contactarse con objetos. Se comprende que en la valoración se destaca la necesidad de más cantidad de actividades prácticas donde se relacionen aspectos teóricos con la realidad. A continuación la respuesta de un estudiante permite ilustrar este aspecto.

“En esta actividad lo que más me gustó fue poder tener contacto con los artefactos e investigarlos desde nuestro punto de vista. En nuestra carrera estamos acostumbrados al exceso de teoría y poca práctica $(A U) "$.

Ampliar conocimientos generales sobre el consumo de energía podría traducirse en ampliar miradas sobre la realidad, animarse a conocer otros temas, enriquecer la cultura general. El hecho de que los estudiantes mencionen que las experiencias les permitieron conocer sobre un tema resulta sumamente valioso desde el punto de vista de la educación. Una crítica que suele realizarse a la formación de grado es la sobre-especialización en un tema, es decir, los pedagogos solo tienen que saber de educación, los ingenieros de física, los biólogos de vegetales y animales, y así. Considerando ciertos estereotipos que circulan sobre qué se debe enseñar y qué no en determinadas clases, resulta importante que los estudiantes destaquen qué aprendieron de una temática que no se relaciona directamente con la formación esperada. A continuación la respuesta de un estudiante permite ejemplificar el aspecto señalado.

“Lo que me pareció más interesante fue romper con algunos mitos respecto del consumo de energía en nuestros propios hogares, la importancia de tener en cuenta cuánto consume un electrodoméstico, cual me conviene a mí y al medioambiente al momento de comprarlo (MC)".

Otro de los aspectos destacados fue hacer algo distinto que rompa con la rutina. En este sentido se mencionó el poder tener experiencias con otros profesionales, la dinámica que tomó la clase, conocer lo que se realiza en otros campos profesionales y conocer sobre mediciones desde otra perspectiva. La respuesta de un estudiante permite ejemplificar el aspecto mencionado.

“Me gustó mucho llevar a cabo esta actividad porque es diferente a las tareas habituales que desarrollamos, porque nos muestra otra forma de considerar las mediciones y porque nos hizo reflexionar sobre una parte de nuestra formación muy importante y la cual no es puesta mucho en práctica a lo largo del cursado de la carrera.

Además la actividad fue favorable en el sentido de que nos permitió tomar más consciencia del consumo que hacemos con nuestros electrodomésticos y reflexionar de ahora en más sobre el uso que hacemos de ellos [...] (AG)".

Tanto en esta como en otras tareas, los estudiantes suelen destacar que les gusta trabajar en grupos. Este tipo de valoraciones resultan interesantes desde el punto de vista de los contextos poderosos, en los que se sugieren los trabajos grupales como modalidad de aprendizaje que permite enriquecer la formación de cada miembro. La respuesta de un estudiante permite ejemplificar el aspecto mencionado. 
“Me gustó trabajar en grupo, discutiendo las características de los elementos, para luego comparar. Fue divertido (PR)".

\section{Aspectos a modificar}

En general los estudiantes no señalaron aspectos a modificar. De 35 estudiantes, 3 mencionaron que modificarían la actividad. Los aspectos a modificar indicados fueron: dedicarle más tiempo a la actividad, hacer más clases como estas e invitar a un psicopedagogo e ingeniero juntos para ir estableciendo relaciones. A continuación las palabras de los estudiantes.

“Tal vez los tiempos no alcanzaron para aprender todo lo que el ingeniero tenía para enseñarnos. Hubiese sido bueno que hubiéramos tenido las cuatro horas seguidas para seguir con la actividad, pero por falta de aula no se pudo (AU)".

“La modificación sería que junto con el ingeniero hubiese estado una psicopedagoga y se mostrara bien lo que una carrera o profesión puede influir o ayudar a la otra (JE)".

“Creo que nada... me pareció una linda experiencia. Solo me gustaría que se aplicaran más estas clases, no solo en esta materia sino en general (LA)".

Los estudiantes hablan de miradas amplias, conocimientos de otras áreas, indisciplinas teóricas y prácticas, rupturas con las rutinas, emociones positivas y trabajo colaborativo. En definitiva, se refieren a las características de los contextos educativos creativos que mencionan autores y especialistas en el área de la creatividad y la educación.

\subsection{Perspectivas de los docentes}

Dos docentes, dos miradas, una propuesta innovadora. Cada uno de los docentes narra las experiencias desde diferentes lugares y perspectivas, haciendo visible el carácter singular e irrepetible de las experiencias (Larrosa, 2006). Sin embargo, ambos confluyen en una idea, una propuesta y una acción educativa: clases inesperadas tanto por un docente invitado como por el docente que de manera inesperada decide invitar.

\subsubsection{Perspectiva del docente esperado que decide invitar}

En el apartado correspondiente compartimos algunos aspectos destacados de la narrativa del docente de la cátedra de Psicometría Educacional que decide invitar a otro docente. Señalamos las experiencias previas y la diversidad de miradas y perspectivas como motor de ideas.

Las experiencias previas como motor de ideas

Todo proceso creativo se inicia con una idea. En la narrativa del docente que decide invitar a un profesor inesperado se observa claramente que la idea emerge de experiencias educativas vivenciadas en diferentes etapas de la formación de grado y postgrado. Lo significativo de dichas experiencias motiva a la docente a generar propuestas inesperadas y a habilitar espacios novedosos para el aprendizaje en la universidad. A continuación, parte de la narrativa del docente.

"La idea surge porque cuando yo era estudiante tenía un profesor que nos sorprendía siempre con las clases. Llegábamos y nos pasaba videos sobre quienes fueron las mujeres científicas, por ejemplo sobre quien fue Marie Curie. Siempre me pareció interesante lo que él hacia porque se ocupaba de la educación en un sentido amplio, al menos eso 
pensaba yo. Siempre nos sorprendía con cosas que no tenían nada que ver con los contenidos de la materia pero que a la larga te enseñaban sobre 'nuevas miradas' o formas diferentes de ver las cosas. Me gustaba porque nos enriquecía culturalmente. Esto fue durante la universidad.

Cuando me recibí, que terminé el doctorado, empecé a dar clase en un secundario y empecé a encontrar muy valioso el hecho de invitar a profesionales capacitados sobre diferentes temáticas que nos comentaran sobre temas diversos. Los chicos no entendían eso y muchas veces me reclamaban que les diera un libro o fotocopia porque no entendían qué tenían que estudiar. También durante ese año salimos mucho a la ciudad, a conocerla. Empecé a darme cuenta de que los chicos aprendían y se cuestionaban cosas que de la forma tradicional -clase magistral- no funcionaba.

Durante la etapa del doctorado anduve mucho conociendo y escuchando gente diversa, participé de teatro, ciclos de cines, charlas, cursos y demás que me siguieron haciendo pensar en que la formación en la universidad no solo debía ocuparse de los temas típicos de las profesiones o carreras sino que, justamente, a veces en las mezclas inesperadas se encontraban grandes puntos para resolver o pensar problemas sociales".

El análisis de la narrativa permite observar que el docente enfatiza la importancia de las relaciones con otras personas y las potencialidades educativas y creativas de dichos vínculos, reconociendo que tienen un fuerte impacto en las ideas y decisiones orientadas a la generación de innovaciones educativas. Los otros juegan un papel importante a la hora de cuestionar las rutinas pedagógicas y proponer alternativas innovadoras. Las ideas, en la innovación educativa y en la creatividad en general, surgen en la interacción con otras personas. Las posibilidades de desarrollarlas también parecen estar condicionadas por dichos vínculos. Los otros y las experiencias vividas con ellos parecen desempeñar un papel importante en las ideas, iniciativas y acciones educativas innovadoras.

La diversidad de miradas y perspectivas como motor de ideas puede observarse en el interés del docente convocante por la educación, la ciencia y la innovación impactando en la invitación de un docente inesperado. Disponer de amplitud en las perspectivas teóricas es indispensable para el desarrollo de innovaciones educativas. Asimismo, es importante generar espacios educativos donde los estudiantes tengan posibilidades de acceder a diferentes miradas y perspectivas sobre conceptos, teorías y prácticas, a veces aparentemente alejados de sus incumbencias profesionales pero que, en definitiva, en la práctica, no lo están.

“Específicamente en el caso del ingeniero la invitación fue porque me interesa la ciencia y pienso que él tiene conocimientos que yo no comprendo y que me podía ayudar. Quizá yo sabía cómo hacer una clase más atractiva pero me faltaba conocer sobre física. A su vez, las materias con números en nuestra carrera suelen ocasionar malestar, pero porque no llegamos a comprender el valor que tienen para resolver problemas de diferente tipo. Entonces pensé que habría sido bueno encontrar un punto de unión entre ambas carreras, pero que a la vez den un plus de cultura general. El punto era medir; los ingenieros miden y nosotros también: ¿qué mide cada uno? y ¿qué problemas se presentan a la hora de medir? Y, a la vez, el tema de la energía era interesante desde el punto de vista del contexto histórico en el que estábamos". 
Además de las perspectivas teóricas, el contexto histórico y las problemáticas referidas al uso de las energías también generan un entorno propicio para reflexionar sobre estas temáticas y su vinculación con la educación en sentido amplio. Un tema, la energía, mirado desde múltiples perspectivas (medición, consumo sustentable, entre otros) ofrece un contexto rico y estimulante para las preguntas, las ideas, las propuestas y las indisciplinas; en definitiva, un ambiente favorable a la creatividad.

\subsubsection{Perspectiva del docente inesperado}

En el presente trabajo se comparten algunos aspectos destacados a partir de la narrativa del docente invitado. Se señalan los desafíos en torno al contexto, el uso de analogías como estrategia de conocimiento y la generación de un clima emocional favorable para aprender.

\section{Contexto inesperado para un docente invitado}

Para el docente invitado el contexto también es inesperado: no sabe muy bien con quiénes se encontrará ni qué le preguntarán. Según sus expresiones, esto genera un importante desafío tanto en la planificación como en el desarrollo de la clase.

“La experiencia de preparar la clase fue un desafío ya que el alumno no está inmerso en un ámbito técnico, por lo que se buscaron ejemplos de la vida diaria. Es importante destacar que en el proceso de búsqueda se encuentran una infinidad de ejemplos sobre energía y potencia. Sin embargo, definir la energía es un concepto muy amplio, considerar a la energía 'como todo aquello que tiene la capacidad de hacer trabajo' decanta en entender que es 'hacer un trabajo' [...]".

El vínculo en un ámbito diferente presenta interrogantes distintos en el transcurso de una clase. Las preguntas que los alumnos realizan suelen ser muy diversas, simples o a veces complejas. Personalmente, estas cuestiones abren otras puertas del conocimiento del tema que se tiene y la capacidad de compartirlo. Es decir que la relación con personas que poseen una formación académica diferente genera un crecimiento personal si se logra la interacción docente-alumno.

Los ámbitos multidisciplinarios son enriquecedores. Desde la organización de la clase y también en el transcurso de la misma, se presentan otros desafíos y otras estructuras, surgen nuevas ideas y se logran otras habilidades. Sin embargo, siempre surgen nuevas preguntas para este tipo de actividades. Por ejemplo, ¿se podría, a través de los experimentos, establecer criterios o características cuantificables sin definirlos previamente? En otras palabras: entender qué capacidad se quiere evaluar de un proceso y si es posible establecer una referencia o patrón para cuantificarlo o cualificarlo sin la inducción de un criterio en especial".

Las clases inesperadas también parecen impactar en la creatividad de los docentes inesperados enriqueciéndolos con nuevas perspectivas y preguntas. Interactuar con otro tipo de estudiantes, con otras experiencias y conocimientos desafía a los docentes inesperados a construir estrategias y propuestas innovadoras de enseñanza, a conjugar planificación e improvisación durante las clases, considerando los aportes y los interrogantes de los estudiantes. Tal como plantean Beghetto y Kaufman (2011), para promover la creatividad en la educación es necesaria cierta improvisación disciplinada. Podríamos agregar también indisciplinada en el sentido de las áreas de conocimientos, 
es decir, como propuestas que las trascienden. Desde diferentes experiencias previas y conocimientos, los estudiantes formulan preguntas y aportes a la clase que interpelan al docente invitado a nuevas perspectivas, ejemplos y explicaciones.

\section{Analogías y construcción de conocimientos}

En la narrativa del docente invitado se destaca el uso de analogías como estrategia de enseñanza y se enfatiza su importancia en los procesos de construcción de conocimientos. También destaca la importancia de los conocimientos previos y las preguntas en el aprendizaje.

“El concepto de medir nos permitió identificar analogías entre la psicometría educacional y la física. Si bien los ingenieros miden la interacción mecánica entre cuerpos, en la psicometría educacional se mide la interacción entre el conocimiento y el alumno. Durante la clase se destacó que en el proceso de medición se compara a la cantidad que se desea medir con una cantidad denominada patrón o referencia. De allí se establecen criterios cuantitativos: menor, igual o mayor que la cantidad denominada patrón. Este proceso es análogo a lo que realizan los psicopedagogos con los test: se busca cuantificar algo, resultado de esa interacción entre la persona y el conocimiento.

Lo más interesante de la clase es cuando los alumnos entienden el concepto de potencia y energía y lo relacionan con lo que saben y empiezan a preguntar como estudiantes de ingeniería..."

Las analogías y las preguntas atraviesan todos los procesos creativos y tal vez sean recursos y herramientas interesantes para la innovación educativa. Construir analogías entre contenidos aparentemente alejados es una acción compleja que demanda procesos cognitivos propios de la creatividad: flexibilidad, pensamiento divergente y combinaciones conceptuales, entre otros. Según Acevedo Díaz (2004) y Sánchez-Ruiz, Romo Santos y Jiménez Jiménez (2013), la construcción de metáforas y analogías es uno de los principales procesos cognitivos involucrados en la creatividad científica. Construir preguntas también es activar procesos cognitivos creativos, "crear implica múltiples dimensiones; pero lo básico, el primer paso en la creatividad es elaborar preguntas" (Corbalán, 2008: 1). En esta línea argumentativa se utiliza la formulación de preguntas como indicador de creatividad en el Test CREA (Corbalán et al., 2015). Destacamos el valor de las analogías y las preguntas como intervenciones pedagógicas orientadas a la creatividad y la innovación en la educación.

\section{La energía de las emociones}

Las emociones positivas mueven los aprendizajes. Tal vez esta sea una buena idea para comprender las innovaciones educativas en unas clases de psicopedagogía sobre energía, electricidad y medición. En los relatos de los docentes y en las experiencias observadas se visualiza un clima emocional óptimo que mueve a los estudiantes a preguntar, participar y hasta a animarse a hacer algunas bromas y jugar.

“Durante la clase fue muy grato observar cómo las chicas se entusiasmaban y preguntaban. Se me hizo un poco largo, la parte teórica, pero fue interesante. Me gustó mucho ver cómo las chicas jugaban, de pronto volvían al jardín de infantes. También me resultó muy interesante la experiencia que comentó Rodolfo como docente y su frustración por no poder cambiar ciertos aspectos de la formación de ingenieros. Ese día se fueron todas contentas y esperando la clase siguiente. En la segunda clase preguntaron cuánto gastaba una pc, compararon los consumos, vieron en qué meses gastaban más y se preguntaron por qué. En fin... a mí me gustó mucho esta experiencia. 
Tenemos pensado volver a hacerla, se me ocurre que estaría bueno mezclar estudiantes de ambas carreras... (Docente esperado que decide invitar)".

Que las emociones desempeñan un papel importante en la educación no parece ser una gran novedad. Sin embargo, en muchos contextos educativos, y en los universitarios en particular, parece ser una cuestión descuidada o poco considerada (Paoloni, 2014). Las experiencias con docentes inesperados han generado entornos emocionalmente propicios para el desarrollo de los aprendizajes. Entusiasmo, risas, alegrías y pasiones puestos en juego tejen una trama interesante para preguntar, construir conocimientos y compartir ideas. En las clases inesperadas se percibía un clima propicio de respeto, alegría, risas y humor, emociones positivas que circulaban y hacían cada vez más fluidas las interacciones dentro del aula.

\section{Consideraciones finales}

Cierta indisciplina óptima es indispensable para construir nuevas perspectivas y acciones educativas. La indisciplina óptima supone vincularse con otros profesionales y otros campos. En esta experiencia se articularon campos diversos como la psicopedagogía y la ingeniería. Pensar una experiencia que tuviera sentido y pudiese ser significada por todos los participantes no fue tarea fácil. En cuanto a los docentes, fue un desafío romper con lo esperado para una clase de psicopedagogía.

Las experiencias y los análisis realizados nos hacen reflexionar respecto de los docentes inesperados. Tal vez sea necesario ampliar la idea inicial desarrollada por Elisondo, Donolo y Rinaudo (2011) y proponer una definición de docente inesperado que incluya, al menos, dos tipologías: docentes inesperados, es decir no esperados por los alumnos, invitados para propuestas específicas y encuadres particulares y docentes esperados que hacen cosas inesperadas como, por ejemplo, invitar a otros docentes y animarse a proponer cosas que rompen con lo que estamos acostumbrados a estudiar en la carrera. Ambos son necesarios para el desarrollo de propuestas como las descritas en el presente artículo. Es indispensable que alguien invite, que alguien decida hacer algo inesperado y también que los docentes inesperados acepten la invitación. Ideas, invitaciones e iniciativas se construyen y reconstruyen en las interacciones entre los docentes esperados (que hacen cosas inesperadas) y los docentes inesperados. Las expresiones de los estudiantes y de los docentes dan cuenta de las potencialidades educativas de las propuestas inesperadas y de las interacciones con docentes invitados. Asimismo, emociones positivas y vivencias significativas desempeñan un papel importante en las experiencias inesperadas y ofrecen interesantes resquicios para la innovación educativa.

Las valoraciones de los estudiantes desafían a realizar más innovaciones. Ellos dicen: "estaría bueno que se hicieran más propuestas de este tipo en las otras asignaturas también". Este tipo de propuestas innovadoras en la universidad contribuye a desarrollar la minicreatividad, es decir, interpretaciones novedosas sobre situaciones, eventos u objetos (Beghetto y Kaufman, 2014). En este sentido es interesante que algunos estudiantes mencionen "si no fuera por esta clase nunca me hubiese puesto a pensar en este tema". Asimismo, y considerando los argumentos neuropsicológicos, la novedad de la propuesta y las emociones subyacentes también contribuyen a que las innovaciones sean recordadas. Por un lado, en futuras implementaciones la propuesta puede ser modificada considerando la solicitud de los estudiantes, en relación a mayor tiempo destinado a la actividad y mayor interacción durante la clase entre los profesionales psicopedagogos e ingeniero, realizando comparaciones explícitas entre los contenidos. Por otro lado, la iniciativa puede modificarse convocando a profesionales de otras ciencias. 


\section{Referencias bibliográficas}

Acevedo Díaz, J. (2004). El papel de las analogías en la creatividad de los científicos: la teoría del campo electromagnético de Maxwell como caso paradigmático de la historia de las ciencias. Revista Eureka sobre enseñanza y divulgación de las ciencias, 1(3): s/d. Recuperado de http://www.redalyc.org/pdf/920/92001304.pdf

Badilla, E. (2007). Descentralizar el aprendizaje: nuevos retos para la educación. Actualidades investigativas en educación, 7 (Especial), 1-27. Recuperado de http://revista.inie.ucr.ac.cr/ index.php/aie/article/view/202

Ballarini, F.; Moncada, D.; Martínez, M.; Alen, N. y Viola, H. (2009) Behavioral tagging is a general mechanism of long-term memory formation. PNAS, 106 (28), 14599-14604. Recuperado de www.pnas.org_cgi_doi_10.1073_pnas.0907078106

Ballarini, F.; Martínez, M.; Díaz Pérez, M.; Moncada, D. y Viola, H. (2013) Memory in elementary school children is improved by an unrelated novel experience. PLOS ONE 8(6), e66875. doi:10.1371/journal.pone.0066875.

Ballarini, F. (2015). REC. Por qué recordamos lo que recordamos y olvidamos lo que olvidamos. Buenos Aires: Sudamericana.

Beghetto R. y Kaufman, J. (2011). Teaching for creativity with disciplined improvisation. In Sawyer, R. (Ed.) Structure and improvisation in creative teaching (pp. 94-109). Cambridge: Cambridge University Press.

Beghetto, R. y Kaufman, J. (2014). Classroom contexts for creativity. High Ability Studies, 25 (1), 53-69.

Bruner, J. (1997). La educación, puerta de la cultura. Colección Aprendizaje. Barcelona: Ed. Visor.

Corbalán Berná, J.; Martínez Zaragoza, F.; Donolo, D.; Alonso Monreal, C.; Tejerina Arreal, M. y Limiñana Gras, M. (2015). CREA. Inteligencia Creativa. Una medida cognitiva de la creatividad. Segunda Edición. Madrid: TEA Ediciones.

Corbalán, J. (2008). ¿De qué se habla cuando hablamos de creatividad? Cuadernos FHyCSUNJu, 35,11-21. Recuperado de http://www.scielo.org.ar/scielo.php?script=sci artt ext\&pid=S1668-81042008000200001

Davies, D.; Jindal-Snape, D.; Collier, C.; Digby, R.; Hay, P. y Howe, A. (2013). Creative learning environments in education: A systematic literature review. Thinking Skills and Creativity, 8, 80-91.

Elisondo, R. (2015). La creatividad como perspectiva educativa. Cinco ideas para pensar los contextos creativos de enseñanza y aprendizaje. Revista Electrónica Actualidades Investigativas en Educación, 15, 3, 1-23. Recuperado de http://revista.inie.ucr.ac.cr/index. php/aie/article/view/855/913 
Elisondo, R. y. Donolo, D. (2013). La lámpara maravillosa y la creatividad. Experiencias en la biblioteca. Creatividad y sociedad, 21, s/d. Recuperado de http://www.creatividadysociedad. com/articulos/21/7.\%20La\%20lampara\%20maravillosa\%20y\%20la\%20creatividad.\%20 Experiencias\%20en\%20la\%20biblioteca.pdf

Elisondo, R. y Donolo, D. (2014). Creatividad y alfabetización informacional. Revista Panorama, 8(15), 23-33. Recuperado de http://dialnet.unirioja.es/descarga/articulo/5051594.pdf

Elisondo, R. y Donolo, D. (2015). Exámenes y sorpresas. Ideas y voces para innovar en evaluación. En: La creatividad como base de la innovación. Ricardo Chrobak y Erika Chrobak (Coords.) Actas del II Congreso Internacional de Investigación y Docencia de la Creatividad (CICREART 2015, Neuquén, Argentina) Recuperado de http://2015presencial.cicreart.com/images/publicaciones/La_creatividad_como_base_de la innovacion_978-987-604-450-9.pdf

Elisondo, R.; Donolo, D. y Rinaudo, M. (2012). Docentes inesperados y creatividad. Experiencias en contextos de educación superior. Revista Electrónica de investigación, docencia y creatividad, 1, 103-114. Recuperado de https://docs.google.com/file/ d/OB5Sju9aeFZ8AN29qNVhlOFlscFU/edit?pli=1

Elisondo, R., Donolo, D. y Rinaudo, M. (2013). The Unexpected and Education: Curriculums for Creativity. Creative Education, 4 (12b). Recuperado de http://www.scirp.org/journal/ce/

Elisondo, R. y Melgar, M. (2015). Museos construidos y reconstruidos. Experiencias educativas para la creatividad. Question, 1(47), 325-341.

Elisondo, R. y Melgar, F. (2016). Las ratas y los estudiantes. El poder de la novedad en la educación. Actas del IV CONGRESO INTERNACIONAL DE CIUDADES CREATIVAS (pp. 314-321). Universidad Complutense de Madrid, 14-16 de enero de 2016. Recuperado de http:// congreso2016.ciudadescreativas.es/Ciudades_Creativas_2016_tomo1.pdf

Elisondo, R.; Rinaudo, M. y Donolo, D. (2011). Actividades inesperadas como oportunidades para la creatividad. Contextos creativos en la Educación Superior Argentina. Revista Innovación Educativa, 11(57), 147-156. Recuperado de http://www.redalyc.org/articulo. oa?id=179422350016

Fernández, A. (2013). Potencia creativa de la alegría. En: Psicología educacional en el contexto de la clínica socioeducativa (pp. 157 -172). Taborda, Alejandra y Leoz, Gladis (Eds.). San Luis: Nueva Editorial Universitaria.

Kozbelt, A.; Beghetto, R. y Runco, M. (2010). Theories of Creativity. En: Kaufman, J. y R. Sternberg. The Cambridge Handbook of Creativity (pp. 447-463). Cambridge: Cambridge Press.

Larrosa, J. (2006). Sobre la experiencia. Aloma, 19, 87-112.

Lucarelli, E. (2009). Teoría y práctica en la universidad. La innovación en las aulas. Buenos Aires: Miño y Dávila Editores.

Manes, F. (2014). Usar el cerebro. Buenos Aires: Planeta. 
Melgar, M. F.; Donolo, D. y Gómez, M. C. (2009). Los museos virtuales y digitales. Aportes desde una perspectiva psicoeducativa. X Encuentro Internacional Virtual Educa Argentina. Organización de los Estados Iberoamericanos (OEI). Buenos Aires: Pontificia Universidad Católica Argentina.

Melgar, M. F. y Donolo, D. (2011). Salir del aula... Aprender de otros contextos: Patrimonio natural, museos e Internet. Revista Eureka sobre enseñanza y divulgación de la ciencia, 8 (3), 323-333. doi: 10498/14396.

Paoloni, P. (2014). El papel de las emociones en los aprendizajes académicos. En: Paola Paoloni, María Cristina Rinaudo y Antonio González (Comps.). Cuadernos de Educación 01. Cuestiones en psicología educacional. Perspectivas teóricas, metodológicas y estudios de campo (pp. 85-131). La Laguna: Sociedad Latina de Comunicación Social. Recuperado de https://issuu.com/revistalatinadecomunicacion/docs/cde01

Pizzolito, A. y Macchiarola, V. (2015). Un estudio sobre cambios planificados en la enseñanza universitaria: origen y desarrollo de las innovaciones educativas. Innovación Educativa, 15 (67):111-134.

Rinaudo, M. (2014). Estudios sobre los contextos de aprendizaje: arenas y fronteras. En: Paola Paoloni, María Cristina Rinaudo y Antonio González (Comps.). Cuadernos de Educación 01.Cuestiones en Psicología Educacional. Perspectivas teóricas, metodológicas y estudios de campo. (pp. 163-205). La Laguna: Sociedad Latina de Comunicación Social. Recuperado de https://issuu.com/revistalatinadecomunicacion/docs/cde01

Rinaudo, M. y Donolo, D. (2000). Casandra y la educación. En Guerci de Siufi, B. Pensando la universidad (pp. 102-130). Jujuy: Editorial UNJU y Red de Editoriales de Universidades Nacionales.

Sánchez-Ruiz, M.; Romo Santos, M. y Jiménez Jiménez, J. (2013). The Role of Metaphorical Thinking in the Creativity of Scientific Discourse. Creativity Research Journal, 25 (4): 361-368.

\section{(Endnotes)}

${ }^{1}$ Múltiples experiencias se construyen ante una propuesta. Docentes y estudiantes viven diferentes experiencias ante un mismo acontecimiento, tal como lo define Larrosa (2006). Por este motivo nos referimos a experiencias en plural. 